\title{
Three Weak Solutions for Nonlocal Fractional Laplacian Equations
}

\author{
Dandan Yang and Chuanzhi Bai \\ Department of Mathematics, Huaiyin Normal University, Huaian, Jiangsu 223300, China \\ Correspondence should be addressed to Chuanzhi Bai; czbai@hytc.edu.cn
}

Received 16 September 2013; Revised 19 November 2013; Accepted 27 November 2013; Published 16 January 2014

Academic Editor: Salvatore A. Marano

Copyright (C) 2014 D. Yang and C. Bai. This is an open access article distributed under the Creative Commons Attribution License, which permits unrestricted use, distribution, and reproduction in any medium, provided the original work is properly cited.

\begin{abstract}
The existence of three weak solutions for the following nonlocal fractional equation $(-\Delta)^{s} u-\lambda u=\mu f(x, u)$ in $\Omega, u=0$ in $\mathbb{R}^{n} \backslash \Omega$, is investigated, where $s \in(0,1)$ is fixed, $(-\Delta)^{s}$ is the fractional Laplace operator, $\lambda$ and $\mu$ are real parameters, $\Omega$ is an open bounded subset of $\mathbb{R}^{n}, n>2 s$, and the function $f$ satisfies some regularity and natural growth conditions. The approach is based on a three-critical-point theorem for differential functionals.
\end{abstract}

\section{Introduction}

In this work we investigate the existence of three weak solutions to the nonlocal counterpart of perturbed semilinear elliptic partial differential equations of the type

$$
\begin{gathered}
-\Delta u-\lambda u=\mu f(x, u) \quad \text { in } \Omega, \\
u=0 \quad \text { in } \mathbb{R}^{n} \backslash \Omega,
\end{gathered}
$$

namely,

$$
\begin{gathered}
(-\Delta)^{s} u-\lambda u=\mu f(x, u) \quad \text { in } \Omega, \\
u=0 \quad \text { in } \mathbb{R}^{n} \backslash \Omega,
\end{gathered}
$$

where $s \in(0,1)$ is fixed, $\Omega$ is a nonempty bounded open subset of $\mathbb{R}^{n}, n>2 s, \lambda$ and $\mu$ are positive real parameters, $f: \Omega \times \mathbb{R} \rightarrow \mathbb{R}$ is a function satisfying suitable regularity and growth conditions, and $(-\Delta)^{s}$ is the fractional Laplace operator defined as

$$
\begin{array}{r}
-(-\Delta)^{s} u(x) \\
:=\mathrm{P} . \mathrm{V} . \int_{\mathbb{R}^{n}} \frac{u(x+y)+u(x-y)-2 u(x)}{|y|^{n+2 s}} d y, \\
x \in \mathbb{R}^{n} .
\end{array}
$$

Fractional Laplace operators have been proved to be valuable tools in the modeling of many phenomena in various fields, such as minimal surfaces, quasi-geostrophic flows, conservation laws, optimization, multiple scattering, anomalous diffusion, ultrarelativistic limits of quantum mechanics, finance, phase transitions, stratified materials, crystal dislocation, semipermeable membranes, flame propagation, soft thin films, and materials science. Recently, there has been significant development in fractional Laplace operators; for examples, see [1-13] and the references therein.

Motivated and inspired by the papers [13-15], in this paper, a variational approach is provided to investigate the existence of three weak solutions to a perturbed nonlocal fractional Laplacian equation (2), by using a three-criticalpoint theorem obtained by Bonanno and Marano in [14].

\section{Preliminaries}

Let $s \in(0,1)$ such that $2 s<n, \Omega \subset \mathbb{R}^{n}$. The classical fractional Sobolev space $H^{s}\left(\mathbb{R}^{n}\right)$ is defined by

$$
H^{s}\left(\mathbb{R}^{n}\right)=\left\{v \in L^{2}\left(\mathbb{R}^{n}\right): \frac{|v(x)-v(y)|}{|x-y|^{(n+2 s) / 2}} \in L^{2}\left(\mathbb{R}^{n} \times \mathbb{R}^{n}\right)\right\}
$$

endowed with the norm (the so-called Gagliardo norm) 


$$
\|v\|_{H^{s}\left(\mathbb{R}^{n}\right)}=\|v\|_{L^{2}\left(\mathbb{R}^{n}\right)}+\left(\iint_{\mathbb{R}^{n} \times \mathbb{R}^{n}} \frac{|v(x)-v(y)|^{2}}{|x-y|^{n+2 s}} d x d y\right)^{1 / 2} .
$$

Let

$$
X_{0}=\left\{g \in H^{s}\left(\mathbb{R}^{n}\right): g=0 \text { a.e. in } \mathbb{R}^{n} \backslash \Omega\right\} .
$$

By [6] in the sequel we can take the function

$$
X_{0} \ni v \longmapsto\|v\|_{X_{0}}=\left(\iint_{\left(\mathbb{R}^{n} \times \mathbb{R}^{n}\right) \backslash \mathcal{O}} \frac{|v(x)-v(y)|^{2}}{|x-y|^{n+2 s}} d x d y\right)^{1 / 2}
$$

as norm on $X_{0}$, where $\mathcal{O}=(\mathscr{C} \Omega) \times(\mathscr{C} \Omega) \subset \mathbb{R}^{n} \times \mathbb{R}^{n}$. It is easily seen that $\left(X_{0},\|\cdot\|_{X_{0}}\right)$ is a Hilbert space, with scalar product

$$
\langle u, v\rangle_{X_{0}}=\iint_{\left(\mathbb{R}^{n} \times \mathbb{R}^{n}\right) \backslash \mathcal{O}} \frac{(u(x)-u(y))(v(x)-v(y))}{|x-y|^{n+2 s}} d x d y .
$$

Since $v \in X_{0}$, we have that the integral in (7) (and in the related scalar product) can be extended to all $\mathbb{R}^{n} \times \mathbb{R}^{n}$.

By a weak solution $u$ of (2) we mean a function $u \in X_{0}$ such that

$$
\begin{gathered}
\iint_{\mathbb{R}^{n} \times \mathbb{R}^{n}} \frac{(u(x)-u(y))(\varphi(x)-\varphi(y))}{|x-y|^{n+2 s}} d x d y \\
\quad-\lambda \int_{\Omega} u(x) \varphi(x) d x \\
=\mu \int_{\Omega} f(x, u(x)) \varphi(x) d x
\end{gathered}
$$

for all $\varphi \in X_{0}$.

Denote by $\lambda_{1}>0$ the first eigenvalue of the operator $(-\Delta)^{s}$ with homogeneous Dirichlet boundary data

$$
\begin{gathered}
(-\Delta)^{s} u=\lambda u \quad \text { in } \Omega, \\
u=0 \quad \text { in } \mathbb{R}^{n} \backslash \Omega .
\end{gathered}
$$

For the existence and the basic properties of $\lambda_{1}$ we may refer to [7]. From [7, 16], we know that if $\lambda<\lambda_{1}$ then we can take a norm on $X_{0}$ as follows:

$$
\begin{gathered}
\|v\|_{X_{0}, \lambda}=\left(\iint_{\left(\mathbb{R}^{n} \times \mathbb{R}^{n}\right) \backslash \mathcal{O}} \frac{|v(x)-v(y)|^{2}}{|x-y|^{n+2 s}} d x d y\right. \\
\left.-\lambda \int_{\Omega}|v(x)|^{2} d x\right)^{1 / 2} .
\end{gathered}
$$

Moreover, we have

$$
m_{\lambda}\|v\|_{X_{0}} \leq\|v\|_{X_{0}, \lambda} \leq M_{\lambda}\|v\|_{X_{0}},
$$

where

$$
m_{\lambda}:=\min \left\{\sqrt{\frac{\lambda_{1}-\lambda}{\lambda_{1}}}, 1\right\}
$$

$$
M_{\lambda}:=\max \left\{\sqrt{\frac{\lambda_{1}-\lambda}{\lambda_{1}}}, 1\right\} .
$$

Remark 1. If $0<\lambda<\lambda_{1}$, then

$$
m_{\lambda}=\sqrt{\frac{\lambda_{1}-\lambda}{\lambda_{1}}}, \quad M_{\lambda}=1 .
$$

Taking into account Lemma 8 in [6], we know that the embedding $j: X_{0} \hookrightarrow L^{\nu}\left(\mathbb{R}^{n}\right)$ is continuous for any $\nu \in$ $\left[1,2^{*}\right]$, while it is compact whenever $v \in\left[1,2^{*}\right)$. Thus, form any $v \in\left[1,2^{*}\right)$ there exists a positive constant $c_{\nu}$ such that

$$
\|v\|_{L^{\nu}\left(\mathbb{R}^{n}\right)} \leq c_{\nu}\|v\|_{X_{0}} \leq c_{\nu} m_{\lambda}^{-1}\|v\|_{X_{0}, \lambda}
$$

for any $v \in X_{0}$.

Let $R:=\sup _{x \in \Omega} \operatorname{dist}(x, \partial \Omega)$; simple calculations show that there is $x_{0} \in \Omega$ such that $B\left(x_{0}, R\right) \subset \Omega$.

Set

$u_{\delta}(x)$

$$
:= \begin{cases}\delta & \text { if } x \in B\left(x_{0}, \frac{R}{2}\right), \\ \frac{2 \delta}{R}\left(R-\left|x-x_{0}\right|\right) & \text { if } x \in B\left(x_{0}, R\right) \backslash B\left(x_{0}, \frac{R}{2}\right), \\ 0 & \text { if } x \in \mathbb{R}^{n} \backslash B\left(x_{0}, R\right) .\end{cases}
$$

Lemma 2. Let $\delta, R>0, s \in(0,1), 0<\lambda<\lambda_{1}$, and let $u_{\delta}$ be defined by (16). Then $u_{\delta} \in X_{0}$, and there exist $C_{*}=$ $C_{*}(n, s, R)>0$ and $C^{*}=C^{*}(n, s, R)$ such that

$$
C_{*} m_{\lambda}^{2} \delta^{2} \leq\left\|u_{\delta}\right\|_{X_{0}, \lambda}^{2} \leq C^{*} \delta^{2}
$$

where $m_{\lambda}$ is as in (14).

Proof. By Proposition 3.4 in [5], we have

$$
\left\|u_{\delta}\right\|_{X_{0}}^{2}=2 C(n, s)^{-1} \int_{\mathbb{R}^{n}}|\xi|^{2 s}\left|\mathscr{F} u_{\delta}(\xi)\right|^{2} d \xi
$$

where

$$
C(n, s)=\left(\int_{\mathbb{R}^{n}} \frac{1-\cos \left(\zeta_{1}\right)}{|\zeta|^{n+2 s}} d \zeta\right)^{-1}
$$

Here $\zeta=\left(\zeta_{1}, \ldots, \zeta_{n}\right)$. From the trivial inequality $|\xi|^{2 s} \leq 1+$ $|\xi|^{2}, s \in(0,1]$, and (18), we obtain

$$
\begin{aligned}
\left\|u_{\delta}\right\|_{X_{0}}^{2} & \leq 2 C(n, s)^{-1} \int_{\mathbb{R}^{n}}\left(1+|\xi|^{2}\right)\left|\mathscr{F} u_{\delta}(\xi)\right|^{2} d \xi \\
& =2 C(n, s)^{-1}\left\|u_{\delta}\right\|_{H^{1}\left(\mathbb{R}^{n}\right)}^{2} .
\end{aligned}
$$


Moreover, according to the definition of norm for $H^{1}\left(\mathbb{R}^{n}\right)$, we get

$$
\begin{aligned}
\left\|u_{\delta}\right\|_{H^{1}\left(\mathbb{R}^{n}\right)}^{2} & =\int_{\mathbb{R}^{n}}\left|\nabla u_{\delta}(x)\right|^{2} d x \\
& =\int_{B\left(x_{0}, R\right) \backslash B\left(x_{0}, R / 2\right)} \frac{(2 \delta)^{2}}{R^{2}} d x \\
& =\frac{4 \delta^{2}}{R^{2}}\left[\operatorname{meas}\left(B\left(x_{0}, R\right)\right)-\operatorname{meas}\left(B\left(x_{0}, \frac{R}{2}\right)\right)\right] \\
& =\frac{4 \delta^{2}}{R^{2}} \frac{\pi^{n / 2}}{\Gamma(1+n / 2)}\left(R^{n}-\left(\frac{R}{2}\right)^{n}\right) \\
& =\frac{4 R^{n-2} \pi^{n / 2}\left(1-1 / 2^{n}\right)}{\Gamma(1+n / 2)} \delta^{2} .
\end{aligned}
$$

By [5], we have

$$
C(n, s)^{-1}=\int_{\mathbb{R}^{n}} \frac{1-\cos \left(\zeta_{1}\right)}{|\zeta|^{n+2 s}} d \zeta=A(n, s) D(s),
$$

where

$$
\begin{gathered}
A(n, s)=\int_{\mathbb{R}^{n-1}} \frac{1}{\left(1+|\eta|^{2}\right)^{(n+2 s) / 2}} d \eta, \\
D(s)=\int_{\mathbb{R}} \frac{1-\cos t}{|t|^{1+2 s}} d t .
\end{gathered}
$$

By polar coordinates, for any $s \in(0,1)$, we obtain

$$
\begin{aligned}
A(n, s)=\omega_{n-2} \int_{0}^{+\infty} \frac{\rho^{n-2}}{\left(1+\rho^{2}\right)^{(n+2 s) / 2}} d \rho \\
=\omega_{n-2}\left[\int_{0}^{1} \frac{\rho^{n-2}}{\left(1+\rho^{2}\right)^{(n+2 s) / 2}} d \rho\right. \\
\left.+\int_{1}^{+\infty} \frac{\rho^{n-2}}{\left(1+\rho^{2}\right)^{(n+2 s) / 2}} d \rho\right] \\
<\omega_{n-2}\left[\int_{0}^{1} \frac{\left(1+\rho^{2}\right)^{(n-2) / 2}}{\left(1+\rho^{2}\right)^{(n+2 s) / 2}} d \rho\right. \\
\left.+\int_{1}^{+\infty} \frac{\rho^{n-2}}{\left(\rho^{2}\right)^{(n+2 s) / 2}} d \rho\right] \\
\left.<\omega_{0}^{1} \frac{d \rho}{1+\rho^{2}}+\int_{1}^{+\infty} \frac{d \rho}{\rho^{2(1+s)}}\right] \\
=\omega_{n-2}\left(\frac{\pi}{4}+\frac{1}{1+2 s}\right)_{:=C_{1}},
\end{aligned}
$$

where $\omega_{n-2}$ is the Lebesgue measure of the unit sphere in $\mathbb{R}^{n-1}$. Furthermore, we have

$$
\begin{aligned}
D(s) & =2 \int_{0}^{+\infty} \frac{1-\cos t}{t^{1+2 s}} d t \\
& =2\left[\int_{0}^{1} \frac{1-\cos t}{t^{1+2 s}} d t+\int_{1}^{+\infty} \frac{1-\cos t}{t^{1+2 s}} d t\right] \\
& <2\left[\int_{0}^{1} \frac{t^{2} / 2}{t^{1+2 s}} d t+2 \int_{1}^{+\infty} \frac{1}{t^{1+2 s}} d t\right] \\
& =\frac{1}{2(1-s)}+\frac{2}{s}:=C_{2} .
\end{aligned}
$$

Thanks to (20)-(25), we conclude that

$$
\left\|u_{\delta}\right\|_{X_{0}}^{2}<\frac{8 R^{n-2} \pi^{n / 2}\left(1-1 / 2^{n}\right)}{\Gamma(1+n / 2)} C_{1} C_{2} \delta^{2}<\infty
$$

which implies that $u_{\delta} \in X_{0}$. By (12), (14), and (26), we obtain

$$
\left\|u_{\delta}\right\|_{X_{0}, \lambda}^{2} \leq\left\|u_{\delta}\right\|_{X_{0}}^{2} \leq C^{*} \delta^{2}
$$

where

$$
C^{*}:=\frac{8 \pi^{n / 2}\left(1-1 / 2^{n}\right)}{\Gamma(1+n / 2)} C_{1} C_{2} R^{n-2} .
$$

Hence, the conclusion of right-hand side of (17) holds.

On the other hand, we have

$$
\begin{aligned}
& \left\|u_{\delta}\right\|_{X_{0}}^{2} \\
& =\iint_{\mathbb{R}^{n} \times \mathbb{R}^{n}} \frac{\left|u_{\delta}(x)-u_{\delta}(y)\right|^{2}}{|x-y|^{n+2 s}} d x d y \\
& =\iint_{B\left(x_{0}, R\right) \times B\left(x_{0}, R\right)} \frac{\left|u_{\delta}(x)-u_{\delta}(y)\right|^{2}}{|x-y|^{n+2 s}} d x d y \\
& \quad+2 \iint_{B\left(x_{0}, R\right) \times\left(\mathbb{R}^{n} \backslash B\left(x_{0}, R\right)\right)} \frac{\left|u_{\delta}(x)-u_{\delta}(y)\right|^{2}}{|x-y|^{n+2 s}} d x d y \\
& =2 \iint_{B\left(x_{0}, R / 2\right) \times\left(B\left(x_{0}, R\right) \backslash B\left(x_{0}, R / 2\right)\right)} \frac{\left|u_{\delta}(x)-u_{\delta}(y)\right|^{2}}{|x-y|^{n+2 s}} d x d y \\
& \quad+\iint_{\left(B\left(x_{0}, R\right) \backslash B\left(x_{0}, R / 2\right)\right) \times\left(B\left(x_{0}, R\right) \backslash B\left(x_{0}, R / 2\right)\right)} \\
& \quad \times \frac{\left|u_{\delta}(x)-u_{\delta}(y)\right|^{2}}{|x-y|^{n+2 s}} d x d y \\
& +2 \iint_{B\left(x_{0}, R\right) \times\left(\mathbb{R}^{n} \backslash B\left(x_{0}, R\right)\right)} \frac{\left|u_{\delta}(x)-u_{\delta}(y)\right|^{2}}{|x-y|^{n+2 s}} d x d y
\end{aligned}
$$




$$
\begin{aligned}
& =\frac{8 \delta^{2}}{R^{2}} \\
& \times \int_{B\left(x_{0}, R / 2\right)}\left(\int_{B\left(x_{0}, R\right) \backslash B\left(x_{0}, R / 2\right)} \frac{|| x-x_{0}|-R / 2|^{2}}{|x-y|^{n+2 s}} d x\right) d y \\
& +\frac{4 \delta^{2}}{R^{2}} \\
& \times \iint_{\left(B\left(x_{0}, R\right) \backslash B\left(x_{0}, R / 2\right)\right) \times\left(B\left(x_{0}, R\right) \backslash B\left(x_{0}, R / 2\right)\right)} \\
& \times \frac{\left\|x-x_{0}|-| y-x_{0}\right\|^{2}}{|x-y|^{n+2 s}} d x d y \\
& +2 \int_{B\left(x_{0}, R / 2\right)}\left(\int_{\mathbb{R}^{n} \backslash B\left(x_{0}, R\right)} \frac{\delta^{2}}{|x-y|^{n+2 s}} d x\right) d y \\
& +\frac{8 \delta^{2}}{R^{2}} \\
& \times \int_{\left(B\left(x_{0}, R\right) \backslash B\left(x_{0}, R / 2\right)\right.}\left(\int_{\mathbb{R}^{n} \backslash B\left(x_{0}, R\right)} \frac{|| y-x_{0}||-\left.R\right|^{2}}{|x-y|^{n+2 s}} d x\right) d y \\
& \geq \frac{8 \delta^{2}}{R^{2}} \\
& \times \int_{B\left(x_{0}, R / 2\right)}\left(\int_{B\left(x_{0}, R\right) \backslash B\left(x_{0}, R / 2\right)} \frac{|| x-x_{0}|-R / 2|^{2}}{|x-y|^{n+2 s}} d x\right) d y \\
& +2 \int_{B\left(x_{0}, R / 2\right)}\left(\int_{\mathbb{R}^{n} \backslash B\left(x_{0}, R\right)} \frac{\delta^{2}}{|x-y|^{n+2 s}} d x\right) d y .
\end{aligned}
$$

For $x \in \mathbb{R}^{n} \backslash B\left(x_{0}, R\right)$ and $y \in B\left(x_{0}, R / 2\right)$, we have

$$
|x-y| \leq\left|x-x_{0}\right|+\left|y-x_{0}\right| \leq\left|x-x_{0}\right|+\frac{R}{2} \leq \frac{3}{2}\left|x-x_{0}\right| .
$$

Thus,

$$
\begin{aligned}
& \int_{B\left(x_{0}, R / 2\right)}\left(\int_{\mathbb{R}^{n} \backslash B\left(x_{0}, R\right)} \frac{1}{|x-y|^{n+2 s}} d x\right) d y \\
& \geq\left(\frac{2}{3}\right)^{n+2 s} \int_{B\left(x_{0}, R / 2\right)}\left(\int_{\mathbb{R}^{n} \backslash B\left(x_{0}, R\right)} \frac{1}{\left|x-x_{0}\right|^{n+2 s}} d x\right) d y \\
& \geq\left(\frac{2}{3}\right)^{n+2 s} \cdot \frac{\omega_{n-1}}{n}\left(\frac{R}{2}\right)^{n} \cdot \omega_{n-1} \int_{R}^{\infty} \frac{\rho^{n-1}}{\rho^{n+2 s}} d \rho \\
& \quad=\left(\frac{2}{3}\right)^{n+2 s} \frac{\omega_{n-1}^{2}}{n}\left(\frac{R}{2}\right)^{n} \cdot \frac{1}{2 s} R^{-2 s}=C_{3} R^{n-2 s},
\end{aligned}
$$

where $C_{3}:=\left(2^{2 s-1} / 3^{n+2 s} n s\right) \omega_{n-1}^{2}$. Moreover, we obtain

$$
\begin{aligned}
& \int_{B\left(x_{0}, R / 2\right)}\left(\int_{B\left(x_{0}, R\right) \backslash B\left(x_{0}, R / 2\right)} \frac{|| x-x_{0}|-R / 2|^{2}}{|x-y|^{n+2 s}} d x\right) d y \\
& \geq \frac{1}{((3 / 2) R)^{n+2 s}} \cdot \frac{\omega_{n-1}}{n}\left(\frac{R}{2}\right)^{n} \\
& \cdot \int_{B\left(x_{0}, R\right) \backslash B\left(x_{0}, R / 2\right)}|| x-x_{0}\left|-\frac{R}{2}\right|^{2} d x \\
&= \frac{2^{2 s} \omega_{n-1}}{n 3^{n+2 s} R^{2 s}} \\
& \times\left[\int_{B\left(x_{0}, R\right)}\left(\left|x-x_{0}\right|^{2}-R\left|x-x_{0}\right|+\frac{R^{2}}{4}\right) d x\right. \\
&= \frac{2^{2 s} \omega_{n-1}}{n 3^{n+2 s} R^{2 s}} \cdot \omega_{n-1} R^{n+2} \\
& \quad \times\left[\frac{n^{2}-n+2}{4 n(n+1)(n+2)}+\frac{1}{2^{n+1} n(n+1)(n+2)}\right] \\
&=C_{4} R^{n+2-2 s},
\end{aligned}
$$

where

$$
C_{4}:=\frac{2^{2 s} \omega_{n-1}^{2}}{n^{2}(n+1)(n+2) 3^{n+2 s}}\left[\frac{n^{2}-n+2}{4}+\frac{1}{2^{n+1}}\right] .
$$

Substitute (31) and (32) into (29), we get

$$
\iint_{\mathbb{R}^{n} \times \mathbb{R}^{n}} \frac{\left|u_{\delta}(x)-u_{\delta}(y)\right|^{2}}{|x-y|^{n+2 s}} d x d y \geq 2\left(C_{3}+4 C_{4}\right) R^{n-2 s} \delta^{2} .
$$

From (34) and (12), we obtain

$$
\left\|u_{\delta}\right\|_{X_{0}, \lambda}^{2} \geq m_{\lambda}^{2}\left\|u_{\delta}\right\|_{X_{0}}^{2} \geq C_{*} m_{\lambda}^{2} \delta^{2},
$$

where

$$
C_{*}:=2\left(C_{3}+4 C_{4}\right) R^{n-2 s} .
$$

Thus, the conclusion of left-hand side of (17) holds.

In this paper our main tool is a three-critical-point theorem of [14] which is recalled below.

Theorem 3 (see [14]). Let $X$ be a reflexive real Banach space; let $\Phi: X \rightarrow \mathbf{R}$ be a coercive, continuously Gâteaux differentiable, and sequentially weakly lower semicontinuous functional whose Gâteaux derivative admits a continuous inverse on $X^{*}$, and let $\Psi: X \rightarrow \mathbf{R}$ be a continuously Gâteaux differentiable functional whose Gâteaux derivative is compact such that

$$
\Phi(0)=\Psi(0)=0 .
$$


Assume that there exist $r>0$ and $\bar{x} \in X$, with $r<\Phi(\bar{x})$, such that

(i) $\sup _{\Phi(x) \leq r} \Psi(x) / r<\Psi(\bar{x}) / \Phi(\bar{x})$;

(ii) for each $\left.\mu \in \Lambda_{r}:=\right] \Phi(\bar{x}) / \Psi(\bar{x}), r / \sup _{\Phi(x) \leq r} \Psi(x)[$ the functional $\Phi-\mu \Psi$ is coercive.

Then, for each $\mu \in \Lambda_{r}$, the functional $\Phi-\mu \Psi$ has at least three distinct critical points in $X$.

\section{Main Result}

Let $f: \Omega \times \mathbb{R} \rightarrow \mathbb{R}$ be a Carathéodory function such that

(H1) there exist $a_{1}, a_{2} \geq 0$ and $q \in\left(1,2_{s}^{*}\right), 2_{s}^{*}:=2 n /(n-2 s)$, such that

$$
|f(x, v)| \leq a_{1}+a_{2}|v|^{q-1}, \quad \forall(x, v) \in \Omega \times \mathbb{R} .
$$

Theorem 4. Let function $f$ satisfy condition (H1). Assume that

(H2) $F(x, v):=\int_{0}^{v} f(x, \tau) d \tau \geq 0$ for all $(x, v) \in \Omega \times \mathbb{R}^{+}$;

(H3) there exist two positive constants $b$ and $p<2$ such that

$$
F(x, v) \leq b\left(1+|v|^{p}\right)
$$

for almost every $x \in \Omega$ and for every $v \in \mathbb{R}$;

(H4) let $0<\lambda<\lambda_{1}$ such that there exist two positive constants $\gamma$ and $\delta$, with $\delta>\sqrt{2 / C_{*}}\left(\gamma / m_{\lambda}\right)$ such that

$$
\frac{\inf _{x \in \Omega} F(x, \delta)}{\delta^{2}}>\frac{b_{1}}{\gamma}+b_{2} \gamma^{q-2}
$$

where

$$
b_{1}=\frac{n 2^{n-1 / 2} a_{1} c_{1} C^{*}}{\omega_{n-1} m_{\lambda} R^{n}}, \quad b_{2}=\frac{n 2^{n+(q-2) / 2} a_{2} c_{q}^{q} C^{*}}{q \omega_{n-1} m_{\lambda}^{q} R^{n}},
$$

and two positive constants $C^{*}$ and $C_{*}$ are as in (28) and (36), respectively. Then, for every $\mu$ belonging to

$$
\Lambda:=] \frac{n 2^{n-1} C^{*}}{\omega_{n-1} R^{n}} \frac{\delta^{2}}{\inf _{x \in \Omega} F(x, \delta)}, \frac{n 2^{n-1} C^{*}}{\omega_{n-1} R^{n}} \frac{1}{b_{1} / \gamma+b_{2} \gamma^{q-2}}[
$$

problem (2) possesses at least three weak solutions in $X_{0}$.

Proof. Let us apply Theorem 3 with $X_{0}$ and

$$
\mathcal{F}_{\lambda, \mu}(u):=\Phi_{\lambda}(u)-\mu \Psi(u), \quad u \in X_{0},
$$

where

$$
\begin{gathered}
\Phi_{\lambda}(u):=\frac{1}{2} \iint_{\mathbb{R}^{n} \times \mathbb{R}^{n}} \frac{|u(x)-u(y)|^{2}}{|x-y|^{n+2 s}} d x d y-\frac{\lambda}{2} \int_{\Omega}|u(x)|^{2} d x, \\
\Psi(u):=\int_{\Omega} F(x, u(x)) d x .
\end{gathered}
$$

For each $u, v \in X_{0}$, one has

$$
\begin{aligned}
& \Phi_{\lambda}^{\prime}(u)(v)= \iint_{\mathbb{R}^{n} \times \mathbb{R}^{n}} \frac{(u(x)-u(y))(v(x)-v(y))}{|x-y|^{n+2 s}} d x d y \\
&-\lambda \int_{\Omega} u(x) v(x) d x, \\
& \Psi^{\prime}(u)(v)=\int_{\Omega} f(x, u(x)) v(x) d x .
\end{aligned}
$$

From the proof of Theorem 1 in [16], we obtain that $\Phi_{\lambda}$ is coercive, continuously Gâteaux differentiable, and sequentially weakly lower semicontinuous functional. Moreover, similar to the proof of proposition in [17], we get by (12) that

$$
\begin{aligned}
& \left(\Phi_{\lambda}^{\prime}(u)-\Phi_{\lambda}^{\prime}(v)\right)(u-v) \\
& =\iint_{\mathbb{R}^{n} \times \mathbb{R}^{n}} \frac{[(u-v)(x)-(u-v)(y)]^{2}}{|x-y|^{n+2 s}} d x d y \\
& \quad-\lambda \int_{\Omega}(u(x)-v(x))^{2} d x \\
& =\|u-v\|_{X_{0}, \lambda}^{2} \geq m_{\lambda}^{2}\|u-v\|_{X_{0}}^{2},
\end{aligned}
$$

for every $u$ and $v$ belonging to $X_{0}$. This actually means that $\Phi_{\lambda}^{\prime}$ is a uniformly monotone operator in $X_{0}$. In addition, standard arguments ensure that $\Phi_{\lambda}^{\prime}$ also turns out to be coercive and hemicontinuos in $X_{0}$. Therefore, $\Phi_{\lambda}^{\prime}$ admits that a continuous inverse in $X_{0}^{*}$ follows immediately by applying Theorem 26. A. of [18]. Furthermore, the functional $\Psi$ is well defined, continuously Gâteaux differentiable with compact derivative and $\Phi_{\lambda}(0)=\Psi(0)=0$.

By [16] we know that being $u$ a weak solution of problem (2) is equivalent to being a critical point of the functional $\mathscr{I}_{\lambda, \mu}$. Since $0<\lambda<\lambda_{1}$, from Lemma 2 , one has

$$
\Phi_{\lambda}\left(u_{\delta}\right)=\frac{1}{2}\left\|u_{\delta}\right\|_{X_{0}, \lambda}^{2} \geq \frac{1}{2} C_{*} m_{\lambda}^{2} \delta^{2}
$$

Bearing in mind that $\delta>\sqrt{2 / C_{*}}\left(\gamma / m_{\lambda}\right)$ (H4), it follows that $\Phi_{\lambda}\left(u_{\delta}\right)>\gamma^{2}$. By $(\mathrm{H} 2)$, we obtain

$$
\begin{aligned}
\Psi\left(u_{\delta}\right) & =\int_{\Omega} F\left(x, u_{\delta}(x)\right) d x \geq \int_{B\left(x_{0}, R / 2\right)} F(x, \delta) d x \\
& \geq \inf _{x \in \Omega} F(x, \delta) \cdot \frac{\omega_{n-1}}{n}\left(\frac{R}{2}\right)^{n} .
\end{aligned}
$$

By Lemma 2, we have

$$
\Phi_{\lambda}\left(u_{\delta}\right)=\frac{1}{2}\left\|u_{\delta}\right\|_{X_{0}, \lambda}^{2} \leq \frac{1}{2} C^{*} \delta^{2} .
$$

So, by (48) and (49), one has

$$
\frac{\Psi\left(u_{\delta}\right)}{\Phi_{\lambda}\left(u_{\delta}\right)} \geq \frac{\omega_{n-1} R^{n}}{n 2^{n-1} C^{*}} \frac{\inf _{x \in \Omega} F(x, \delta)}{\delta^{2}} .
$$


Thanks to $(\mathrm{H} 1)$, one has

$$
F(x, u) \leq a_{1}|u|+\frac{a_{2}}{q}|u|^{q}, \quad(x, u) \in \Omega \times \mathbb{R} .
$$

Thus, by (15) and (51), for every $u \in X_{0}: \Phi_{\lambda}(u) \leq r$, we obtain

$$
\begin{aligned}
\Psi(u) & =\int_{\Omega} F(x, u(x)) d x \leq a_{1}\|u\|_{L^{1}(\Omega)}+\frac{a_{2}}{q}\|u\|_{L^{q}(\Omega)}^{q} \\
& \leq \frac{\sqrt{2} a_{1} c_{1}}{m_{\lambda}} \sqrt{r}+\frac{2^{q / 2} c_{q}^{q} a_{2}}{q m_{\lambda}^{q}} r^{q / 2} .
\end{aligned}
$$

Therefore

$$
\sup _{\left.\left.u \in \Phi_{\lambda}^{-1}(]-\infty, r\right]\right)} \Psi(u) \leq \frac{\sqrt{2} a_{1} c_{1}}{m_{\lambda}} \sqrt{r}+\frac{2^{q / 2} c_{q}^{q} a_{2}}{q m_{\lambda}^{q}} r^{q / 2} .
$$

Denote the function

$$
\chi(r):=\frac{\sup _{\left.\left.u \in \Phi_{\lambda}^{-1}(]-\infty, r\right]\right)} \Psi(u)}{r}, \quad r>0 .
$$

By (53), we have

$$
\chi(r) \leq \frac{\sqrt{2} a_{1} c_{1}}{m_{\lambda}} r^{-1 / 2}+\frac{2^{q / 2} c_{q}^{q} a_{2}}{q m_{\lambda}^{q}} r^{q / 2-1}, \quad r>0 .
$$

Owing to (50), (55), and (H4), we have

$$
\begin{aligned}
\chi\left(\gamma^{2}\right) & \leq \frac{\sqrt{2} a_{1} c_{1}}{m_{\lambda} \gamma}+\frac{2^{q / 2} c_{q}^{q} a_{2}}{q m_{\lambda}^{q}} \gamma^{q-2}=\frac{\omega_{n-1} R^{n}}{n 2^{n-1} C^{*}}\left(\frac{b_{1}}{\gamma}+b_{2} \gamma^{q-2}\right) \\
& <\frac{\omega_{n-1} R^{n}}{n 2^{n-1} C^{*}} \frac{\inf _{x \in \Omega} F(x, \delta)}{\delta^{2}} \leq \frac{\Psi\left(u_{\delta}\right)}{\Phi_{\lambda}\left(u_{\delta}\right)} .
\end{aligned}
$$

Hence, the assumption (i) of Theorem 3 is satisfied.

Furthermore, if $p<2$, for each $u \in X_{0},|u|^{p} \in L^{2 / p}(\Omega)$, Hölder's inequality and (15) give

$$
\begin{aligned}
\int_{\Omega}|u(x)|^{p} d x & \leq\|u\|_{L^{2}(\Omega)}^{p} \operatorname{meas}(\Omega)^{(2-p) / 2} \\
& \leq \frac{c_{2}^{p}}{m_{\lambda}^{p}}\|u\|_{X_{0}, \lambda}^{p} \operatorname{meas}(\Omega)^{(2-p) / 2}, \quad \forall u \in X_{0} .
\end{aligned}
$$

Due to (H3) and (57), we deduce that

$$
\begin{aligned}
\mathscr{F}_{\lambda, \mu}(u) \geq & \frac{1}{2}\|u\|_{X_{0}, \lambda}^{2}-\frac{\mu b c_{2}^{p}}{m_{\lambda}^{p}} \operatorname{meas}(\Omega)^{(2-p) / 2}\|u\|_{X_{0}, \lambda}^{p} \\
& -\mu b \cdot \operatorname{meas}(\Omega), \quad \forall u \in X_{0} .
\end{aligned}
$$

Hence, $\mathscr{J}_{\lambda, \mu}(u)$ is a coercive functional for every positive parameter $\mu$, in particular, for each $\mu \in \Lambda \subset] \Phi_{\lambda}\left(u_{\delta}\right) / \Psi\left(u_{\delta}\right)$, $\gamma^{2} / \sup _{\Phi_{\lambda}(u) \leq \gamma^{2}} \Psi(u)$ [. So also condition (ii) holds. So all the assumptions of Theorem 3 are satisfied. Thus, for each $0<$ $\lambda<\lambda_{1}$ there exists $\mu>0$, depending on $\lambda$, such that, for any $\mu \in \Lambda$, the functional $\mathscr{J}_{\lambda, \mu}(u)$ has at least three distinct critical points that are weak solutions to problem (2).
Remark 5. Similar to Example 3.1 in [15], we can give a concrete example of function satisfying hypotheses (H1)(H4). Set $q \in\left(2,2_{s}^{*}\right), s \in(0,1)$, and $0<p<2$ and let

$$
h:=\max \left\{1, \frac{1}{m_{\lambda}} \sqrt{\frac{2}{C_{*}}},\left(A_{1}+A_{2}\right)^{1 /(q-2)} q^{1 /(q-2)}\right\},
$$

where

$$
A_{1}=\frac{n 2^{n-1 / 2} c_{1} C^{*}}{\omega_{n-1} m_{\lambda} R^{n}}, \quad A_{2}=\frac{n 2^{n+(q-2) / 2} c_{q}^{q} C^{*}}{q \omega_{n-1} m_{\lambda}^{q} R^{n}} .
$$

From (H4) we know that $b_{1}=a_{1} A_{1}$ and $b_{2}=a_{2} A_{2}$. Let $r$ be a positive constant such that $r>h$ and consider the following continuous and positive function $f: \Omega \times \mathbb{R} \rightarrow \mathbb{R}$ :

$$
f(x, v):= \begin{cases}1+|v|^{q-1} & \text { if } v \leq r \\ 1+r^{q-p} v^{p-1} & \text { if } v>r .\end{cases}
$$

Obviously, $f(x, v) \leq 1+|v|^{q-1}$ for each $(x, v) \in \Omega \times \mathbb{R}$, and (H1) holds. Furthermore, for every $\eta \in \mathbb{R}$, we have

$$
F(x, \eta) \leq\left(r+\frac{r^{q}}{p}\right)\left(1+|\eta|^{\max \{1, p\}}\right) .
$$

Thus the conditions $(\mathrm{H} 2)$ and $(\mathrm{H} 3)$ are satisfied. Moreover, $r>h \geq\left(1 / m_{\lambda}\right) \sqrt{2 / C_{*}}$ and

$$
\frac{\int_{0}^{r} f(x, t) d t}{r^{2}}=\frac{r^{q-2}}{q}+\frac{1}{r}>A_{1}+A_{2},
$$

which implies that (H4) holds.

\section{Conflict of Interests}

The authors declare that there is no conflict of interests regarding the publication of this paper.

\section{Acknowledgments}

The authors thank the referees for their valuable and helpful suggestions and comments that improved the paper. This work is supported by Natural Science Foundation of Jiangsu Province (BK2011407) and Natural Science Foundation of China (11271364 and 10771212).

\section{References}

[1] L. A. Caffarelli, S. Salsa, and L. Silvestre, "Regularity estimates for the solution and the free boundary of the obstacle problem for the fractional Laplacian," Inventiones Mathematicae, vol. 171, no. 2, pp. 425-461, 2008.

[2] L. Silvestre, "Regularity of the obstacle problem for a fractional power of the Laplace operator," Communications on Pure and Applied Mathematics, vol. 60, no. 1, pp. 67-112, 2007.

[3] X. Cabré and J. Tan, "Positive solutions of nonlinear problems involving the square root of the Laplacian," Advances in Mathematics, vol. 224, no. 5, pp. 2052-2093, 2010. 
[4] X. Cabré and Y. Sire, "Nonlinear equations for fractional Laplacians I: regularity, maximum principles, and Hamiltonian estimates," Annales de l'Institut Henri Poincare C, 2013.

[5] E. Di Nezza, G. Palatucci, and E. Valdinoci, "Hitchhiker's guide to the fractional Sobolev spaces," Bulletin des Sciences Mathématiques, vol. 136, no. 5, pp. 521-573, 2012.

[6] R. Servadei and E. Valdinoci, "Mountain pass solutions for nonlocal elliptic operators," Journal of Mathematical Analysis and Applications, vol. 389, no. 2, pp. 887-898, 2012.

[7] R. Servadei and E. Valdinoci, "Variational methods for nonlocal operators of elliptic type," Discrete and Continuous Dynamical Systems A, vol. 33, no. 5, pp. 2105-2137, 2013.

[8] R. Servadei and E. Valdinoci, "Lewy-Stampacchia type estimates for variational inequalities driven by (non)local operators," Revista Matemática Iberoamericana, vol. 29, no. 3, pp. 1091-1126, 2013.

[9] M. M. Fall and T. Weth, "Nonexistence results for a class of fractional elliptic boundary value problems," Journal of Functional Analysis, vol. 263, no. 8, pp. 2205-2227, 2012.

[10] F. Ferrari and I. E. Verbitsky, "Radial fractional Laplace operators and Hessian inequalities," Journal of Differential Equations, vol. 253, no. 1, pp. 244-272, 2012.

[11] R. Servadei and E. Valdinoci, "The Brezis-Nirenberg result for the fractional Laplacian," Transactions of the American Mathematical Society. In press.

[12] C. Bai, "Existence results for non-local operators of elliptic type," Nonlinear Analysis A, vol. 83, pp. 82-90, 2013.

[13] S. Dipierro, G. Palatucci, and E. Valdinoci, "Existence and symmetry results for a Schrödinger type problem involving the fractional Laplacian," Le Matematiche, vol. 68, no. 1, pp. 201-216, 2013.

[14] G. Bonanno and S. A. Marano, "On the structure of the critical set of non-differentiable functions with a weak compactness condition," Applicable Analysis, vol. 89, no. 1, pp. 1-10, 2010.

[15] G. Bonanno and G. Molica Bisci, "Three weak solutions for elliptic Dirichlet problems," Journal of Mathematical Analysis and Applications, vol. 382, no. 1, pp. 1-8, 2011.

[16] G. Molica Bisci and R. Servadei, "A bifurcation result for nonlocal fractional equations," Journal of Applied Analysis. In press.

[17] G. Bonanno and P. Candito, "Three solutions to a Neumann problem for elliptic equations involving the p-Laplacian," Archiv der Mathematik, vol. 80, no. 4, pp. 424-429, 2003.

[18] E. Zeidler, Nonlinear Functional Analysis and Its Applications, vol. 2, Springer, New York, NY, USA, 1985. 


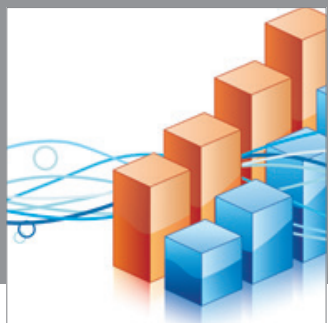

Advances in

Operations Research

mansans

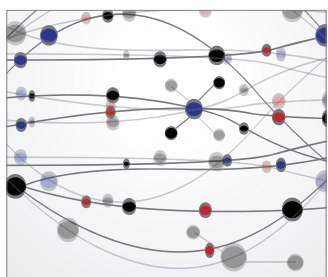

The Scientific World Journal
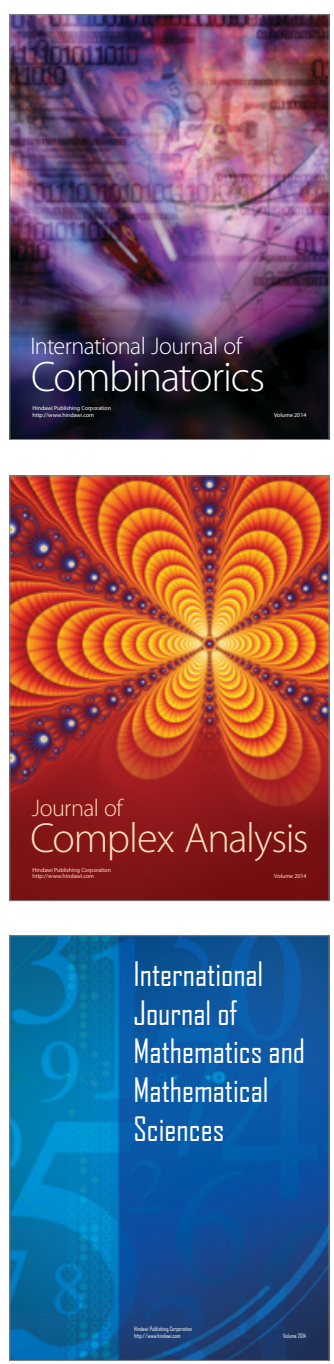
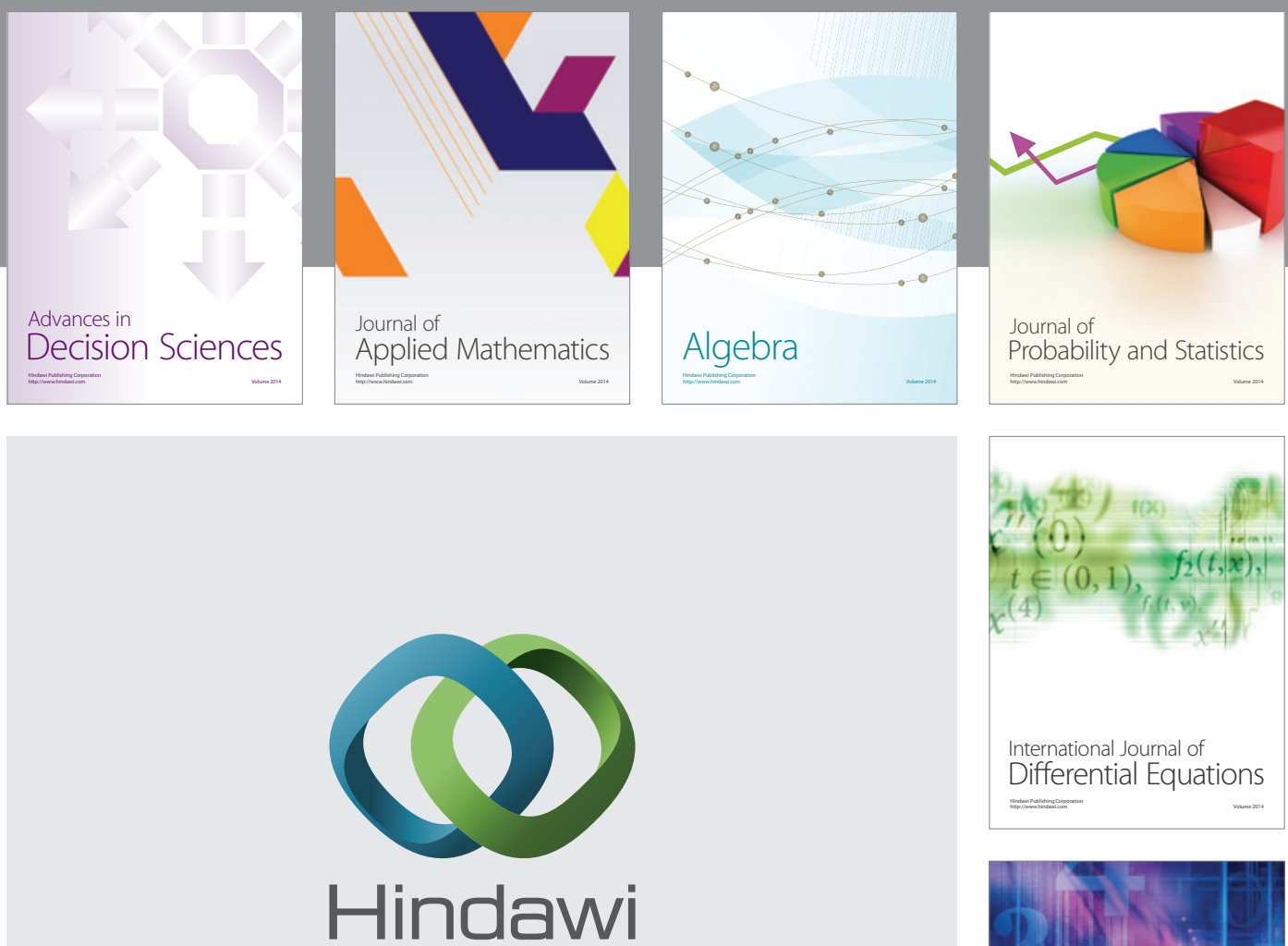

Submit your manuscripts at http://www.hindawi.com
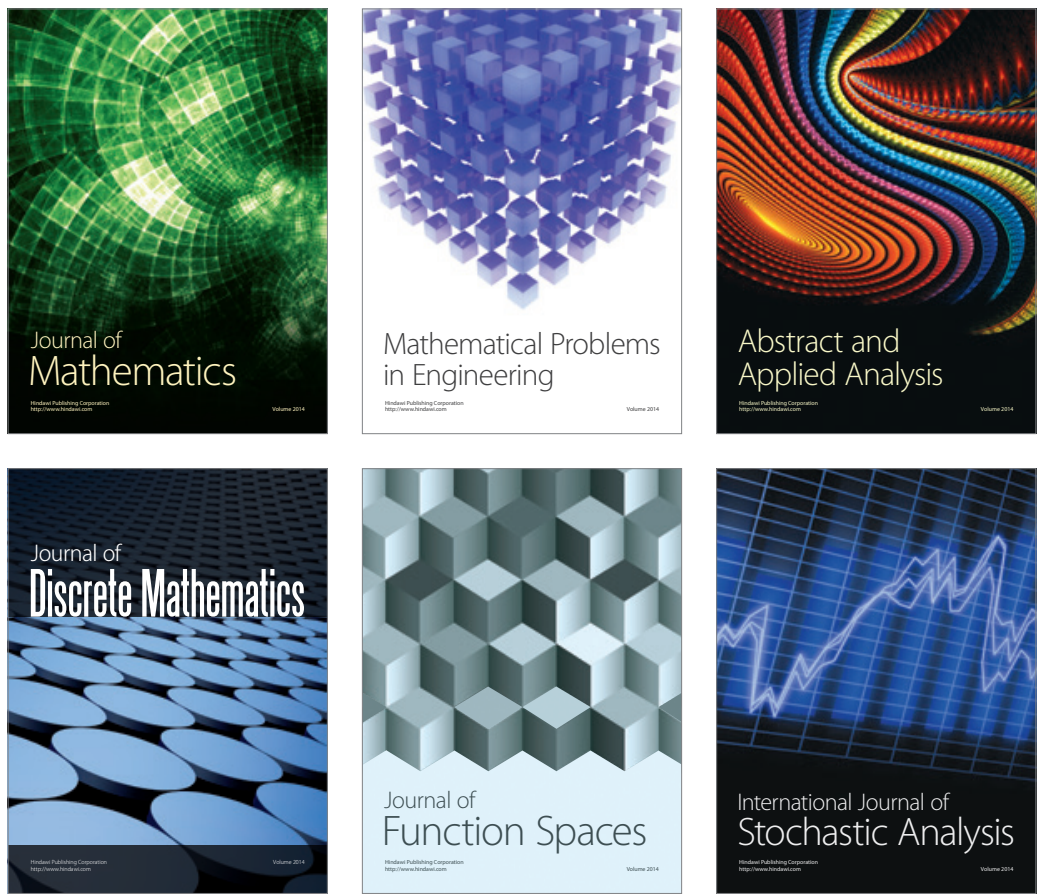

Journal of

Function Spaces

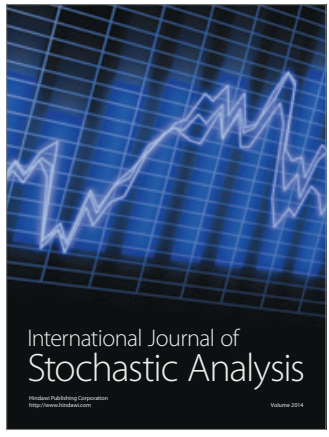

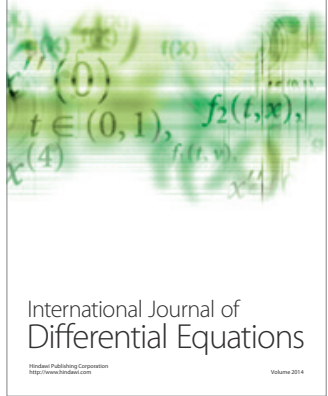
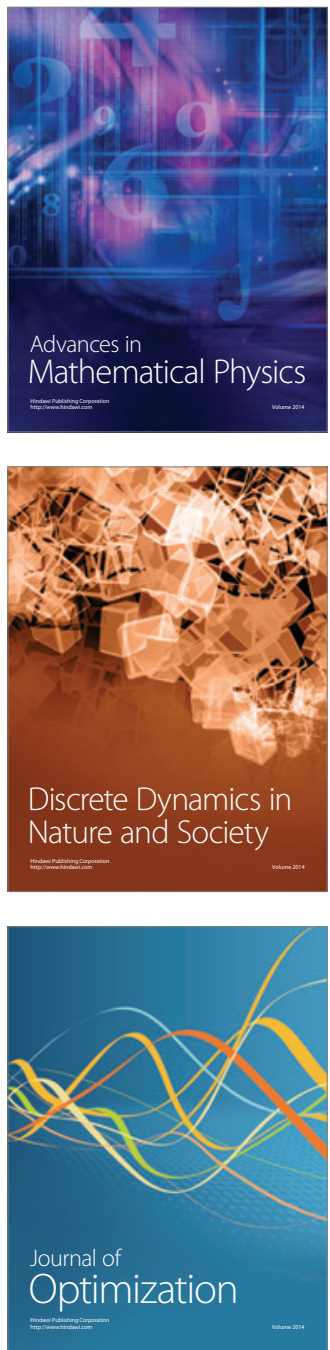